\title{
On holomorphic one-forms transverse to closed hypersurfaces
}

\author{
TOSHIKAZU ITO ${ }^{1}$ and BRUNO SCÁRDUA ${ }^{2}$ \\ ${ }^{1}$ Department of Natural Science, Ryukoku University, Fushimi-ku, Kyoto 612, Japan \\ ${ }^{2}$ Instituto de Matemática, Universidade Federal do Rio de Janeiro, \\ Caixa Postal 68530, 21945-970 Rio de Janeiro, RJ, Brasil
}

Manuscript received on May 26, 2003; accepted for publication on June 3, 2003; presented by MARCIO SOARES

\begin{abstract}
In this note we announce some achievements in the study of holomorphic distributions admitting transverse closed real hypersurfaces. We consider a domain with smooth boundary in the complex affine space of dimension two or greater. Assume that the domain satisfies some cohomology triviality hypothesis (for instance, if the domain is a ball). We prove that if a holomorphic one form in a neighborhood of the domain is such that the corresponding holomorphic distribution is transverse to the boundary of the domain then the Euler-Poincare-Hopf characteristic of the domain is equal to the sum of indexes of the one-form at its singular points inside the domain. This result has several consequences and applies, for instance, to the study of codimension one holomorphic foliations transverse to spheres.
\end{abstract}

Key words: Holomorphic one-form, vector field, Euler-Poincaré characteristic, foliation, distribution.

\section{INTRODUCTION}

The classical theorem of Poincaré-Hopf (Milnor 1965) implies that for a smooth (real) vector field $X$ defined in a neighborhood of the closed ball $\overline{B^{2 n}(0 ; R)} \subset \mathbb{R}^{2 n}$ and transverse to the boundary $\partial B^{2 n}(0, R)=S^{2 n-1}(0 ; R)$ there is at least one singular point $p \in \operatorname{sing}(X) \cap B^{2 n}(0 ; R)$. Moreover, if the singularities of $X$ in $B^{2 n}(0 ; R)$ are isolated then $\sum \operatorname{Ind}(X ; p)=1$ where $p$ runs through all the singular points $p \in \operatorname{sing}(X) \cap B^{2 n}(0 ; R)$ and $\operatorname{Ind}(X ; p)$ is the index of $X$ at the singular point $p$. In (Ito 1994) one can find a version of this theorem for holomorphic vector fields on $\mathbb{C}^{n}$. This motivated the study of codimension one holomorphic foliations on open subsets of $\mathbb{C}^{n}$ with the

Mathematics Subject Classification: 32S65; 57R30.

Correspondence to: Bruno Scárdua

E-mail: scardua@im.ufrj.br 
transversality property with real submanifolds and, particularly, the case of foliations transverse to spheres $S^{2 n-1}(0 ; R) \subset \mathbb{C}^{n}$ (see [Ito and Scardua 2002a] for more information). Let us recall the notion of transversality we shall use:

Given a holomorphic one form $\Omega$ in $U \subset \mathbb{C}^{n}$ for each $p \in U$ with $\Omega(p) \neq 0$ we define a $(n-1)$-dimensional linear subspace $P_{\Omega}(p):=\left\{v \in T_{p}\left(\mathbb{C}^{n}\right) ; \Omega(p) \cdot v=0\right\}$. If $\Omega(p)=0$ we set $P_{\Omega}(p):=\left\{O_{p}\right\}<T_{p}\left(\mathbb{C}^{n}\right)$ and we shall say that the distribution $P_{\Omega}$ defined by $\Omega$ is singular at $p$. As usual we assume that $\operatorname{cod} \operatorname{sing}(\Omega) \geq 2$ so that if $\Omega$ is integrable i.e., $\Omega \wedge d \Omega \equiv 0$ in $U$ (equivalently if $P_{\Omega}$ is integrable) then $P_{\Omega}=T \mathcal{F}_{\Omega}$ for a unique singular holomorphic foliation $\mathcal{F}_{\Omega}$ of codimension one in $U$ having as singular set $\operatorname{sing}\left(\mathcal{F}_{\Omega}\right)=\operatorname{sing}\left(P_{\Omega}\right)=\operatorname{sing}(\Omega)$. Including the non-integrable case we have the following definition of transversality.

Definition 1. (Ito and Scárdua 2002a). Given a smooth (real) submanifold $M \subset U$ we shall say that $P_{\Omega}$ is transverse to $M$ if for every $p \in M$ we have $T_{p} M+P_{\Omega}(p)=T_{p}\left(\mathbb{R}^{2 n}\right)$ as real linear spaces.

In particular, since $P_{\Omega}(p)=\{0\}$ for any singular point $p$, we conclude that $\operatorname{sing}\left(P_{\Omega}\right) \cap M=\varnothing$ if $\operatorname{dim}_{\mathbb{R}} M<\operatorname{dim}_{\mathbb{R}} U$. We also point out that if $\Omega$ is a holomorphic integrable one-form in $U$ then given a submanifold $M \subset U$ the distribution $P_{\Omega}$ is transverse to $M$ if, and only if, for each $p \in M$ we have $p \notin \operatorname{sing}\left(\mathcal{F}_{\Omega}\right)$ and also $T_{p}\left(L_{p}\right)+T_{p}(M)=T_{p}\left(\mathbb{R}^{2 n}\right)$ (as real linear spaces); where $L_{p}$ is the leaf of $\mathcal{F}_{\Omega}$ that contains the point $p \in M$. Thus $P_{\Omega}$ is transverse to $M$ if, and only if, the foliation $\mathcal{F}_{\Omega}$ defined by $\Omega$ is transverse to $M$ in the sense of (Ito and Scárdua 2002a) which is the ordinary sense.

Let now $\Omega=\sum_{j=1}^{n} f_{j}(z) d z_{j}$ in holomorphic coordinates in a neighborhood of the closed domain $\bar{D}$ in $\mathbb{C}^{n}$, then $\operatorname{sing}(\Omega)=\left\{p ; f_{j}(p)=0, \forall j\right\}$ and we define the gradient of $\Omega$ as the complex $C^{\infty}$ vector field

$$
\operatorname{grad}(\Omega)=\sum_{j=1}^{n} \overline{f_{j}(z)} \frac{\partial}{\partial z_{j}} .
$$

Given any isolated singularity $p \in \operatorname{sing}(\Omega)$ we define the index of $\Omega$ at $p$ by

$$
\operatorname{Ind}(\Omega ; p)=\operatorname{Ind}\left(\sum_{j=1}^{n} f_{j}(z) \frac{\partial}{\partial z_{j}} ; p\right)=\operatorname{Ind}(\overline{\operatorname{grad}(\Omega)} ; p) \text {. }
$$

Our main result is the following:

THEOREM 1. (Ito and Scárdua 2002a,b). Let $D \subset \subset \mathbb{C}^{n}$ be a relatively compact domain with smooth boundary $\partial D \subset \mathbb{C}^{n}$. Assume that the (canonical) exact sequence $H^{1}(D, \mathbb{Z}) \rightarrow H^{1}(\partial D, \mathbb{Z}) \rightarrow 0$ is exact. Then given any holomorphic one-form $\Omega$ in a neighborhood $U$ of $\bar{D}$ in $\mathbb{C}^{n}$ such that the corresponding holomorphic distribution $P_{\Omega}$ is transverse to the boundary $\partial D$ we have

$$
\sum_{p \in \operatorname{sing}(\Omega) \cap D} \operatorname{Ind}(\Omega ; p)=(-1)^{n} \chi(D)
$$


where $\chi(D)$ is the Euler-Poincaré-Hopf characteristic of $D$.

Write $\Omega=\sum_{j=1}^{n} f_{j}(z) d z_{j}$ in holomorphic coordinates in a neighborhood of $o$ in $\mathbb{C}^{n}$. We shall say that $o$ is a simple singularity of $\Omega$ if:

$$
\left\{\begin{array}{l}
f_{j}(o)=0, \quad j=1, \ldots, n \\
\operatorname{Det}\left(\frac{\partial f_{j}(o)}{\partial z_{k}}\right)_{j, k=1}^{n} \neq 0 .
\end{array}\right.
$$

As an immediate consequence of Theorem 1 we obtain:

Theorem 2. (Ito and Scárdua 2002a). Let $\Omega$ be a holomorphic one-form in a neighborhood $U$ of the closed ball $\overline{B^{2 n}(0 ; R)}$ in $\mathbb{C}^{n}, n \geq 2$. Assume that $P_{\Omega}$ is transverse to the sphere $S^{2 n-1}(0 ; R)=$ $\partial B^{2 n}(0 ; R)$. Then $n$ is even and $\Omega$ has exactly one singular point $o \in \overline{B^{2 n}(0 ; R)}$. Moreover this singular point is simple.

In (Ito and Scárdua 2002b) one finds a natural extension of the above result for holomorphically embedded closed balls in Stein spaces. In case $D \subset \subset \mathbb{C}^{n}$ is Stein and $n \geq 3$ we also obtain:

Corollary 1. (Ito and Scárdua 2002b). Let $D \subset \mathbb{C}^{n}$ be a relatively compact Stein domain with smooth boundary $\partial D \subset \mathbb{C}^{n}$ and suppose $n \geq 3$. Given any holomorphic one-form $\Omega$ in a neighborhood of $\bar{D}$ with $P_{\Omega}$ transverse to $\partial D$ we have

$$
\sum_{p \in \operatorname{sing}(\Omega) \cap D} \operatorname{Ind}(\Omega ; p)=(-1)^{n} \chi(D) .
$$

Since $\operatorname{Ind}(\Omega ; p) \geq 1$ for all (isolated) singular point we obtain:

Corollary 2. Let $D \subset \subset \mathbb{C}^{n}$ and $\Omega$ be as in Theorem 1. If $\chi(D)=0$ then $\operatorname{sing}(\Omega) \cap \bar{D}=\emptyset$. If $\chi(D) \geq 1$ then $\operatorname{sing}(\Omega) \cap D \neq \emptyset$ and necessarily $n$ is even.

We also refer to (Ito and Scárdua 2002c) for further results.

\section{SKETCH OF THE PROOF OF THEOREM 1}

We have the canonical exact sequence $H^{1}(D) \rightarrow H^{1}(\partial D) \rightarrow H^{2}(D, \partial D)$ and by hypothesis $H^{1}(D) \rightarrow H^{1}(\partial D) \rightarrow 0$ is exact. Take a holomorphic vector field $\vec{n}$ in a neighborhood of $\bar{D}$ such that for each $q \in \partial D$ the vector $\vec{n}(q)$ is non-zero and ortogonal to the complex tangent space $T_{q}^{\mathbb{C}}(\partial D)<T_{q}\left(\mathbb{C}^{n}\right)$. Given $\Omega$ as in Theorem 1 we introduce the analytic set

$$
\Sigma_{\Omega}:=\{q ; \Omega(q) \cdot \vec{n}(q)=0\} .
$$

Then for each $q \in \partial D$ we have $q \in \Sigma_{\Omega}$ if and only if $\operatorname{grad}(\Omega)(q) \in T_{q}^{\mathbb{C}}(\partial D)$. Since the vector field $\operatorname{grad}(\Omega)$ is orthogonal to $P_{\Omega}$ we conclude that there exists a smooth bump-function $\varphi: \mathbb{C}^{n} \stackrel{C^{\infty}}{\longrightarrow} \mathbb{R}$ such that

$$
Z:=\operatorname{grad}(\Omega)+\varphi \cdot \vec{n} .
$$


is transverse to $\partial D$.

Using the hypothesis that $H^{1}(D) \rightarrow H^{1}(\partial D) \rightarrow 0$ is exact we obtain a real smooth section (ie. a $C^{\infty}$ real vector field) $\widetilde{\xi} \in T Z$ over a neighborhood of $\bar{D}$ which is transverse to $\partial D$; indeed $\widetilde{\xi}$ is obtained as extension of a suitable vector field $\xi(z)=a(z) X(z)+b(z) Y(z)$ defined in a neighborhood of $\partial D$ and which is transverse to $\partial D$, where $X$ and $Y$ are given by

$$
Z=\frac{1}{2}(X-\sqrt{-1} Y)
$$

Theorem 1 now follows from Poincaré-Hopf Index theorem (Milnor 1965) applied to the vector field $\widetilde{\xi}$ once we have the following lemma:

LEMMA 1. (Ito and Scárdua 2002a). In the above situation we have:

(i) $\operatorname{sing}(\Omega) \cap D$ is finite, $\operatorname{sing}(\Omega) \cap D=\operatorname{sing}(\widetilde{\xi})$;

(ii) Given any $p \in \operatorname{sing}(\Omega) \cap D$ we have $\operatorname{Ind}(\Omega ; p)=(-1)^{n} \operatorname{Ind}(\widetilde{\xi} ; p)$.

Theorem 2 is a straightforward consequence of Theorem 1 . Corollary 1 is proved recalling that by Poincaré-Lefschetz duality (Griffiphs and Harris 1978) we have that $H^{2}(D, \partial D) \simeq H_{2 n-2}(D)=$ 0 in the case of a Stein domain and $n \geq 3$.

\section{ACKNOWLEDGMENT}

We are grateful to Professor N. Kawazumi for his interest and valuable suggestions in improving our original results.

\section{RESUMO}

Nesta nota anunciamos alguns resultados obtidos no estudo de distribuições holomorfas admitindo hipersuperfícies reais fechadas transversais. Consideramos um domínio com bordo suave no espaço afim complexo de dimensão dois ou maior. Suponha que o domínio satisfaz uma certa hipótese de trivialidade cohomológica (por exemplo, se o domínio é uma bola). Provamos que se uma um-forma holomorfa em uma vizinhança do domínio é tal que a distribuição holomorfa correspondente é transversal ao bordo do domínio então a característica de Euler-Poincaré-Hopf do domínio é igual à soma dos índices da um-forma nos seus pontos singulares dentro do domínio. Este resultado tem várias conseqüências e se aplica, por exemplo, ao estudo de folheações holomorfas de codimensão um transversais a esferas.

Palavras-chave: Um-forma holomorfa, campo de vetores, característica de Euler-Poincaré, folheação, distribuição.

\section{REFERENCES}

Griffiths P AND Harris J. 1978. Principles of Algebraic Geometry; John Wiley \& Sons, N.Y. 
Iто T. 1994. A Poincaré-Bendixson Type Theorem for Holomorphic Vector Fields; RIMS publication, Kyoto, nำ 878.

Ito T And ScÁRduA B. 2002a. A Poincaré-Hopf type theorem for holomorphic 1-forms; Pre-publication Ryukoku University, Kyoto.

Ito T AND ScÁRdUA B. 2002b. On complex distributions transverse to spheres; submitted.

ITo T AND SCÁRDUA B. 2002c. On real transverse sections of holomorphic foliations; submitted.

MiLnOR J. 1965. Topology from the differential view point, The University Press of Virginia, Charlottesville. 